\title{
First-principles study of the effect of charge on the stability of a diamond nanocluster surface
}

\author{
Noejung Park, ${ }^{1,2 *}$ Sungil Park, ${ }^{3}$ Nong-Moon Hwang, ${ }^{3}$ Jisoon Ihm,,${ }^{4,5}$ Syogo Tejima, ${ }^{2}$ and Hisashi Nakamura ${ }^{2}$ \\ ${ }^{1}$ Computational Science and Engineering Laboratory, Samsung Advanced Institute of Technology, P.O. Box 111, Suwon 440-660, Korea \\ ${ }^{2}$ Research Organization for Information Science and Technology, 2-2-54, Naka-Meguro, Meguro-ku, Tokyo 153-0061, Japan \\ ${ }^{3}$ Center for Microstructure Science of Materials, School of Materials Science \& Engineering, Seoul National University, \\ Seoul 151-742, Korea \\ ${ }^{4}$ Center for Nanotube and Nanostructured Composites, Sungkyunkwan University, Suwon 440-746, Korea \\ ${ }^{5}$ School of Physics, Seoul National University, Seoul 151-742, Korea \\ (Received 26 September 2003; revised manuscript received 22 January 2004; published 20 May 2004)
}

\begin{abstract}
Effects of net charge on the stability of the diamond nanocluster are investigated using the first-principles pseudopotential method with the local density approximation. We find that the charged nanocluster favors the diamond phase over the reconstruction into a fullerene-like structure. Occupying the dangling bond orbitals in the outermost surface, the excess charge can stabilize the bare diamond surface and destabilize the C-H bond on the hydrogenated surface. In combination with recent experimental results, our calculations suggest that negative charging could promote the nucleation and further growth of low-pressure diamond.
\end{abstract}

DOI: 10.1103/PhysRevB.69.195411

PACS number(s): 61.46.+w, 64.70.Nd, 81.07.-b

\section{INTRODUCTION}

Synthetic diamonds have been considered to be an important material in designing electronic devices and in mechanical coating because of hardness, chemical stability, and high thermal conductivity. ${ }^{1-4}$ While the high pressure condition is widely accepted as a sufficient condition for stabilizing the diamond phase over graphite, reports on diamond synthesis under low pressure conditions have cast doubts on the conventional growth mechanism.,

Although the presence of atomic hydrogen has been considered to be a crucial factor in the low pressure synthesis of diamond, ${ }^{1,3,7-9}$ understandings on the exact role of hydrogen atoms are rather ambiguous. One point of view has claimed that atomic hydrogen etches selectively the nondiamond carbon phase or cleans the growing diamond surface providing reactive sites for further growth. ${ }^{1}$ In another point of view, it was suggested that adsorbed hydrogen atoms stabilize the diamond surface over the reconstruction into $s p^{2}$-bonded graphitic layers. ${ }^{710}$ However, recent reports on the successful synthesis of diamond without atomic hydrogen ${ }^{11,12}$ suggested the existence of an unknown microscopic mechanism. Especially, as it appears that particles formed in the vapor phase are negatively charged, ${ }^{5,13}$ the effect of charges on the nucleation stage of diamond particles becomes more intriguing than ever. In addition, a similar question extends to the biasenhanced nucleation on the substrate. ${ }^{14-16}$

In this work, we perform first-principles density functional calculations to investigate the effects of a net electric charge on the stability of diamond particles. In order to calculate the charged unit cell without a spurious long-ranged interaction, we employ the formulation of the spherically cutoff Coulomb interaction. Our calculations show that a negative charge can stabilize the diamond surfaces from reconstructing into graphitic layers as does atomic hydrogen. Moreover, the strength of $\mathrm{C}-\mathrm{H}$ bonding at the surface is substantially reduced under the presence of the negative charge, which explains why the diamond formation under the dis- charge condition ${ }^{5}$ is almost independent of hydrogen partial pressure.

\section{COMPUTATIONAL METHODS}

The effective potential for Kohn-Sham ${ }^{17}$ equation can be written as

$$
V_{\mathrm{eff}}(\mathbf{r})=\sum_{\lambda} \frac{-Z_{\lambda}}{\left|\mathbf{r}-\mathbf{R}_{\lambda}\right|}+\int \frac{\rho\left(\mathbf{r}^{\prime}\right)}{\left|\mathbf{r}-\mathbf{r}^{\prime}\right|} d^{3} \mathbf{r}^{\prime}+v_{\mathrm{xc}}[\rho(\mathbf{r})]
$$

On the right-hand side are the ionic potential $\left(V_{\text {ion }}\right)$, the electron-electron Coulomb potential $\left(V_{H}\right)$, and the exchange-correlation potential terms, respectively. The ionic potential $\left(V_{\text {ion }}\right)$ is described by the norm-conserving pseudopotential generated through the Troullier-Martins scheme ${ }^{18}$ and its nonlocal part is converted into the fully separable form of Kleinman and Bylander. ${ }^{19}$ The Ceperley-Alder form of the local density approximation is employed for the exchange-correlation potential. ${ }^{20,21}$ A common practice has been the evaluation of the potentials in the reciprocal lattice space. ${ }^{22}$ The electron-electron Coulomb potential can exactly be written as $V_{H}(\mathbf{G})=4 \pi \rho(\mathbf{G}) / \mathbf{G}^{2}$ in the reciprocal space. If the system is neutral, the long-ranged $1 / r$ tails in the first and second terms of Eq. (1) cancel each other and the potential term does not diverge in the limit of $\mathbf{G}=\mathbf{0}$. However, if the system is charged, the long-ranged tail survives and the periodic boundary condition induces spurious Coulomb interactions.

In order to calculate the Coulomb potential of the charged cluster using the efficiency of the fast fourier transform (FFT), we employ the Onida's formulation of the spherically cutoff Coulomb interaction ${ }^{23,24}$

$$
V_{H}(\mathbf{r})=\int \zeta\left(\mathbf{r}, r^{\prime}\right) \rho\left(\mathbf{r}^{\prime}\right) d^{3} \mathbf{r}^{\prime}
$$

where 

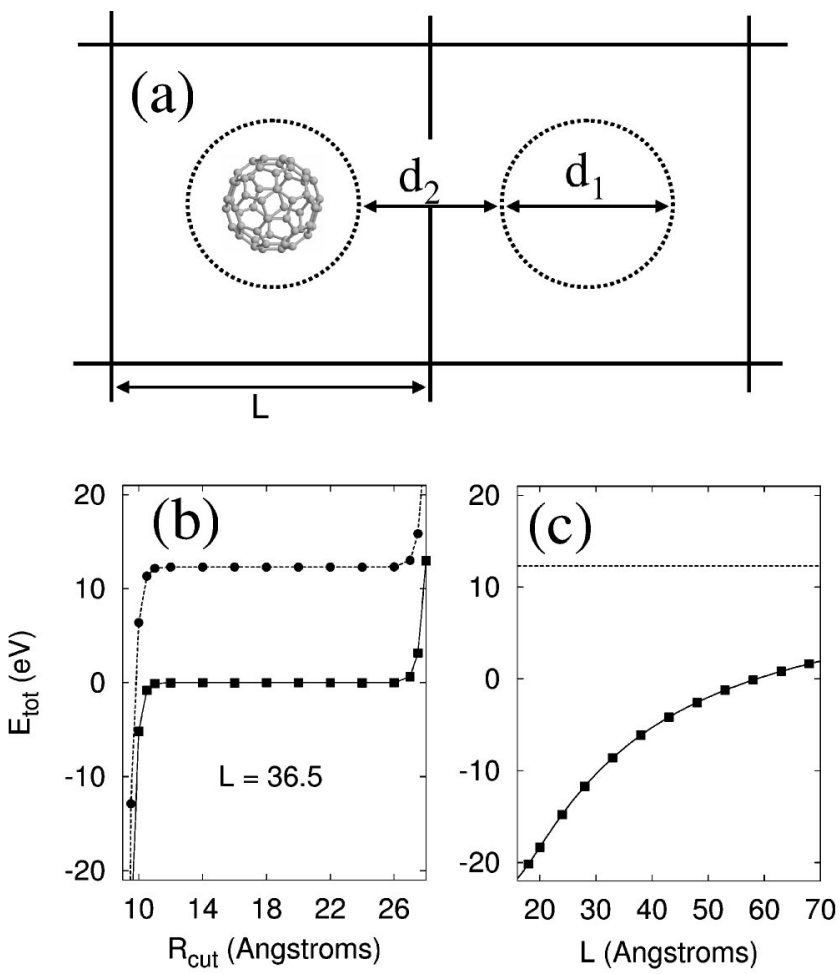

FIG. 1. (a) $\mathrm{A} \mathrm{C}_{60}$ in the cubic supercell of side $L$. The sphere containing all the charge within the unitcell should have the diameter $d_{1} \geqslant D_{f}+2 R_{b}$, where $D_{f}$ is the diameter of $\mathrm{C}_{60}$ and $R_{b}$ the range of the basis orbital. (b) The total energy results for the chargeneutral and the negatively charged $\mathrm{C}_{60}$ by the cutoff Coulomb interaction method with various $R_{\text {cut }}$ 's. Solid squares are for the charge-neutral case and the solid spheres are for the charged case with six electrons. The lines are only for visual convenience. Here the supercell dimension is fixed to $L=36.5 \AA$. (c) The total energy results (solid squares) for the $\mathrm{C}_{60}$ charged with six electrons by conventional supercell method with various supercell dimensions $(L)$. The solid line is the least square fit to the functional form suggested by Makov and Payne for the charged cluster submerged in the jellium (Ref. 27). The dotted line is the total energy in the limit of the infinitely large $L$. Total energies in the ordinate of (b) and (c) are with respect to that of the charge neutral case.

$$
\zeta\left(\mathbf{r}, \mathbf{r}^{\prime}\right)= \begin{cases}\frac{1}{\left|\mathbf{r}-\mathbf{r}^{\prime}\right|} & \text { for }\left|\mathbf{r}-\mathbf{r}^{\prime}\right|<R_{\text {cut }}, \\ 0 & \text { otherwise. }\end{cases}
$$

Now the Fourier component of the Coulomb potential is exactly $V_{H}(\mathbf{G})=\left[4 \pi \rho(\mathbf{G}) / \mathbf{G}^{2}\right]\left[\mathbf{1}-\cos \left(|\mathbf{G}| R_{\text {cut }}\right)\right]$, which is finite in the limit of $\mathbf{G}=0$. The matrix element for the ionic potential is evaluated in the real space grid without periodic images.

Here we explicitly verify the efficiency of the cutoff Coulomb interaction formula by performing the total energy calculation for the charge-neutral as well as the negatively charged $\mathrm{C}_{60}$. The minimal basis set is employed here and the range of the basis orbital $\left(R_{b}\right)$ is generated to be $6 r_{\mathrm{Bohr}}$ $(\approx 3.175 \AA) .{ }^{25}$ Figure 1 (a) shows the cubic supercell of side $L$ and the $\mathrm{C}_{60}$ therein. The whole charge density of the $\mathrm{C}_{60}$ in the unitcell can be defined within the sphere of diameter $d_{1}$ $=D_{f}+2 R_{b} \approx 13.2 \AA$, where the $D_{f}$ is the diameter of the $\mathrm{C}_{60}$. In order to include all the Coulomb interaction within the supercell and in order to exclude any Coulomb interaction between the neighboring cells, the cutoff radius $R_{\text {cut }}$ should be larger than $d_{1}$ and smaller than $d_{2}$. Figure 1(b) shows the total energy results with variable $R_{\text {cut }}$ while the supercell dimension is fixed to $L=36.5 \AA$. The total energies in the ordinate are with respect to that of the charge-neutral $\mathrm{C}_{60}$. As noted in the Ref. 27, the spurious interaction between neighboring cells is strictly avoided whenever $R_{\text {cut }}$ satisfies the aforementioned criterion, $13.2 \AA \leqslant R_{\text {cut }} \leqslant 23.3 \AA$. Noticeably the total energies in Fig. 1(b) readily converges even if the $R_{\text {cut }}$ deviates from the above range by $2 \AA$ or so. This is obviously because the valence charge density of each carbon atom is mostly within the $2 \AA$ from the atom center, ${ }^{26}$ and thereby the dominant contribution to the electronic Coulomb interaction for $\mathrm{C}_{60}$ can virtually be evaluated by letting $d_{1} \approx D_{f}+2 \times 2 \AA \approx 11 \AA$. The same system charged with six electrons is calculated with the conventional periodic boundary condition and the compensating jellium charge, as shown in Fig. 1(c). The least square fit to the functional form suggested by Makov et al. ${ }^{27}$ results in the $E_{\text {tot }}(L)-E_{\text {tot }}^{0}$ $=12.260-733.018 / L+48325.832 / L^{3}$, where $E_{\text {tot }}^{0}$ is the total energy for the charge-neutral cluster. The value of the total energy in the infinitely large supercell shows a good consistency with that calculated using the cutoff Coulomb interaction.

In the remaining part of this paper, we use the double numeric basis $\operatorname{set}^{28}$ for the localized pseudoatomic orbitals, which is generated through the method of Sankey and Niklewski. ${ }^{26}$ The supercell dimension and the cutoff radius for Coulomb potential is taken as $L=42 \AA, R_{\text {cut }}=21 \AA$ for the calculation presented in Figs. 2 and 3. A slightly smaller $L$ and $R_{\text {cut }}$ are used for Fig. 5. The minimum energy configuration is obtained through the relaxation of the atomic force which includes the Pulay-like correction ${ }^{29}$ to the HellmanFeynman force.

\section{RESULTS}

Figure 2(a) shows the model geometry used in the calculation. From the bulk diamond structure in which the $\mathrm{C}-\mathrm{C}$ bond length is set to $1.53 \AA$, the carbon cluster of $\mathrm{C}_{165}$ in the shape of octahedron is excised. All of its eight faces are diamond (111) surfaces. Six carbon atoms at each corner of the $\mathrm{C}_{165}$ octahedron would have only two nearest neighbors. ${ }^{10}$ By removing these six atoms at the corner, all the outermost carbon atoms in the model geometry $\left(\mathrm{C}_{159}\right)$ have three nearest neighbors as shown in Fig. 2(a). Through the geometry optimization calculations, the model geometry of Fig. 2(a) is transformed to a partially graphitized structure shown in Fig. 2(b). Consistent with the recent works on bucky diamond, ${ }^{30}$ the outermost surface shows a significant reconstruction. The interior consisting of 35 carbon atoms retains $s p^{3}$ geometry with 18 disconnected bonds from the outer surface. Apart from the twelve atoms represented by the darker spheres in Fig. 2(b), the surface atoms show mainly $s p^{2}$-bonding geometry. The twelve atoms are connected to the interior structure with a bond length elongated by $\sim 0.15 \AA$ compared with that in a perfect $s p^{2}$ region. 


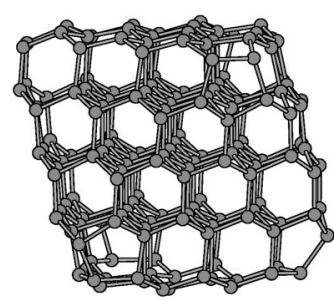

(a)

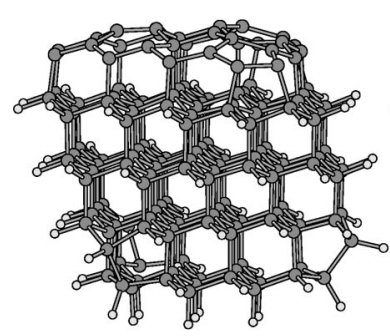

(c)

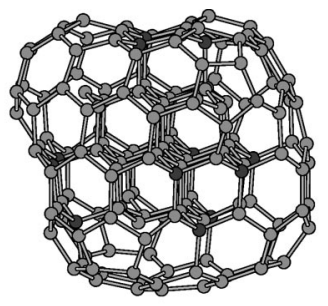

(b)

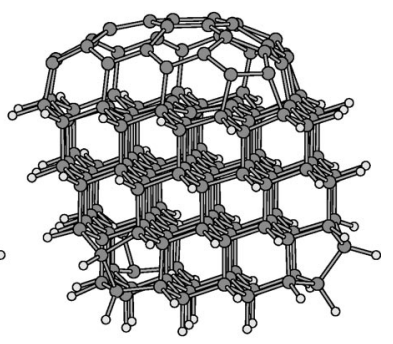

(d)
FIG. 2. Model geometries of diamond nanoclusters. (a) Octahedron-shaped $\mathrm{C}_{159}$ cluster excised from the bulk diamond. (b) Optimized geometry of (a). (c), (d) Optimized geometries of (a) whose seven faces are saturated with hydrogen atoms. Gray spheres in (a) -(d) and white spheres in (c) and (d) represent carbon and hydrogen atoms, respectively. Darker spheres in (b) denote 12 carbon atoms in the outer surface preserving the connection with the interior structure.

Here, we only show the result for the $\mathrm{C}_{159}$ cluster. However, the relaxation behavior of the $\mathrm{C}_{165}$ cluster is very similar to this and is consistent with literature. ${ }^{10}$

Experiments $^{31,32}$ have shown that heating or irradiation ${ }^{33}$ induces a transformation from diamond nanoparticle to graphitic onion structure. Although the structure shown in Fig. 2(b) is not a perfect onion structure, this relaxation result is believed to reflect the pathway in such transformation. Here we note that, in addition to the graphitization, a complete saturation of the surface dangling bonds by atomic hydrogens could also be an obstacle to the growth of the diamond particle. Earlier works ${ }^{7}$ showing that a hydrogen covered dia-

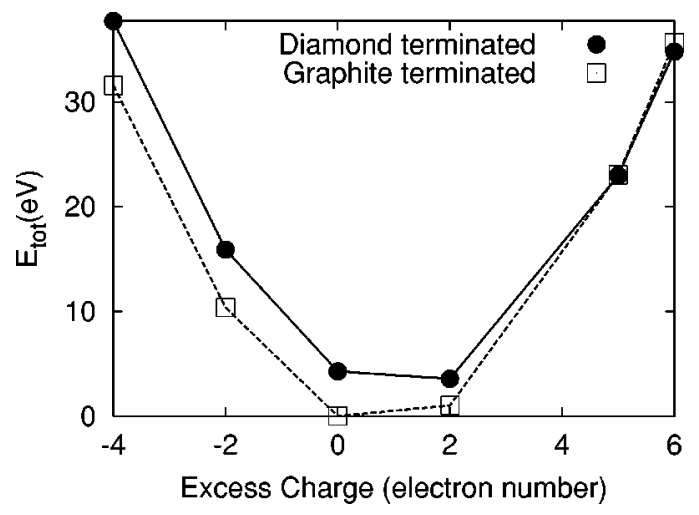

FIG. 3. Total energy of diamond terminated and graphite terminated cases with respect to various charging states. The abscissa is the excess charge in electron numbers and the ordinate is total energy with respect to that of charge-neutral graphite terminated case.

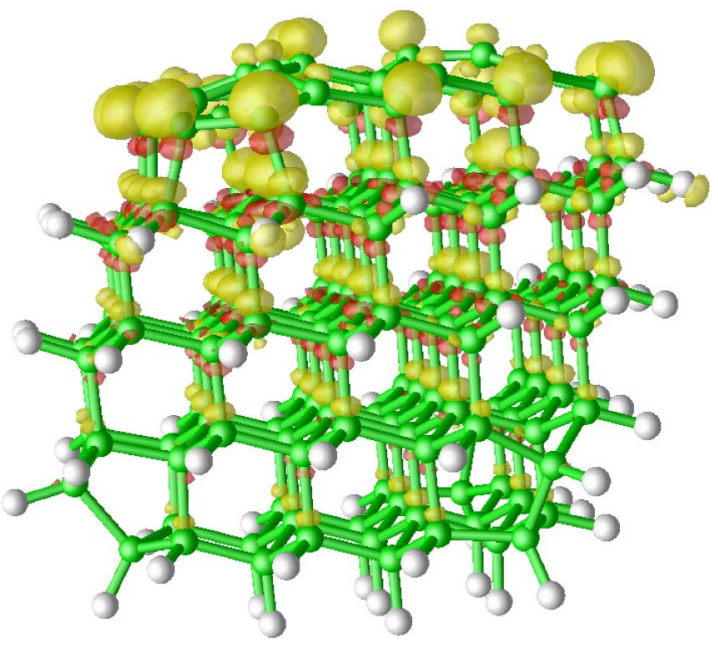

FIG. 4. The distribution of excess charge of the six electroncharged diamond terminated case. Gray balls and white balls are carbon atoms and hydrogen atoms, respectively. The dark lobes in the upper part of the figure are an isosurface of the excess charge density.

mond nanoparticle is more stable than graphitic fragments does not appear to explain the nucleation of diamond during the low pressure synthesis. The ubiquitous existence of the diamondoid structures in natural petroleum, which is a diamond structure consisting of a few tens of carbon and hydrogen atoms, ${ }^{34,35}$ implies that the full coverage of hydrogen atoms should lead to the stopping of the growth of diamond.

To study the effect of charging on the surface of a diamond nanoparticle, we passivate the seven faces of the model geometry with hydrogen atoms while dangling bonds remain unsaturated in one face. Figures 2(c) and 2(d) show optimized geometries in the charge-neutral case. While a significant number of dangling bonds are attached to the outermost atoms in Fig. 2(c), equivalent atoms in Fig. 2(d) show an almost complete reconstruction into a fullerenelike structure. Noting that the surface of Fig. 2(c) is not so much deviated from the original diamond structure, we designate it as diamond terminated. Whereas, Fig. 2(d) is denoted as graphite terminated. Obviously in neutral case, the graphite terminated structure is favored over diamond terminated.

However, as we increase the number of excess electrons in the cluster, the graphite terminated structure becomes less and less favored. In Fig. 3 we plot the total energy of two structures as a function of excess charge, with respect to that of the charge-neutral graphite terminated case. The relative stability between graphite terminated and diamond terminated cases is reversed when the excess charge is five electrons. Obviously, the excess charge populates and stabilizes the dangling bonds. In Fig. 4, we show the distribution of the excess electrons for the diamond terminated case by substraction of the electron density of the neutral case from that of the six excess electrons case.

In this work, we follow the constant-charge mode ${ }^{36}$ to compare the stability between the diamond terminated and graphite terminated case. Though the relative stability between differently charged states could not be accounted for solely by the total energy shown in Fig. 3, the energy differ- 

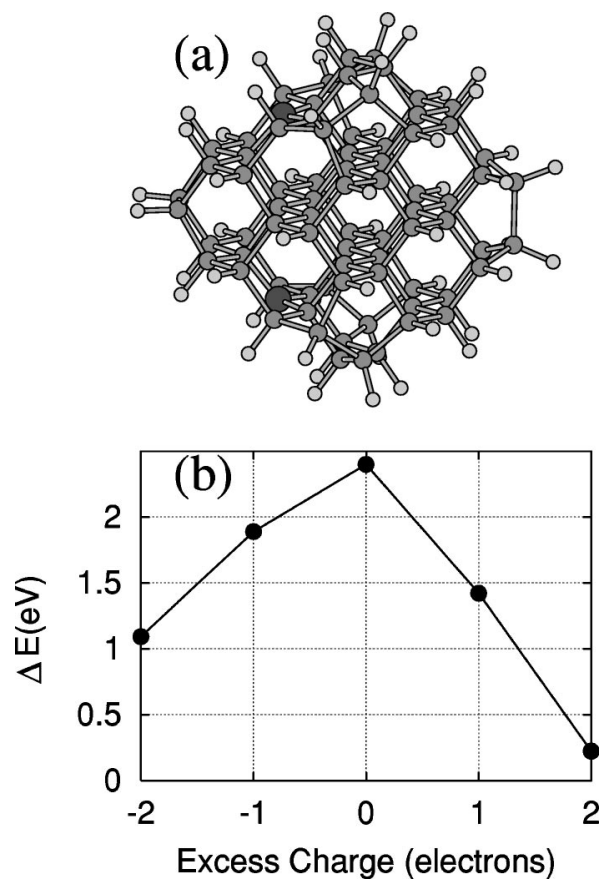

FIG. 5. (a) $\mathrm{C}_{78} \mathrm{H}_{52}$ cluster with two missing hydrogen atoms. Gray spheres represent carbon atoms and white spheres represent hydrogen atoms. Larger darker spheres emphasize carbon atoms in the hydrogen-free sites. (b) The $\mathrm{C}-\mathrm{H}$ binding energy with respect to $\mathrm{C}_{78} \mathrm{H}_{50}$ and hydrogen molecule. The abscissa is the excess charge in electron numbers.

ence between the different morphology with a given negative charge is believed to explain the low pressure synthesis of the diamond particle under electron discharge condition. At this time, the amount of charges for the diamond particle in the vapor phase of the realistic situation is hardly measurable. A measurable structural changes of the carbon nanostructures upon charging of $-10 e \sim+20 e$ has also studied ${ }^{37}$ in different context.

Now we investigate the $\mathrm{C}-\mathrm{H}$ bond strength at the diamond surface with variable charging states. As shown in Fig. 5, the diamond cluster of 78 carbon atoms are passivated by 50 hydrogen atoms leaving two dangling bonds unsaturated. We measure the $\mathrm{C}-\mathrm{H}$ binding energy on the surface by calculating the value $\Delta E^{(n)}=\frac{1}{2}\left\{E_{\text {tot }}^{(n)}\left(\mathrm{C}_{78} \mathrm{H}_{50}\right)+E_{\text {tot }}\left(\mathrm{H}_{2}\right)\right.$ $\left.-E_{\text {tot }}^{(n)}\left(\mathrm{C}_{78} \mathrm{H}_{52}\right)\right\}$, where the superscript $n$ represents the number of excess electrons and $\mathrm{C}_{78} \mathrm{H}_{52}$ represents the struc- ture fully covered by hydrogen atoms. The atomic force of each geometry is relaxed down to $10^{-3} \mathrm{Ry} / a_{0}$. The negatively charging ( $n=1, n=2)$ weakens the $\mathrm{C}-\mathrm{H}$ bond significantly and the $\Delta E$ becomes negligible. The charge density plot (not shown) shows that the excess charge occupies the dangling bond orbitals, thereby stabilizing the surface. The optimized geometry also shows that the negatively charged cases preserve most of the original $s p^{3}$ bond, meanwhile reconstructions in neutral and positively charged cases force carbon atoms at hydrogen-free sites to adopt a $s p^{2}$-like geometry with their three neighbors.

We note that with given small shift of the Fermi level, $\mathrm{C}_{78} \mathrm{H}_{50}$ is more likely to accommodate excess electrons than $\mathrm{C}_{78} \mathrm{H}_{52}$. Thus, although we follow the constant-charge mode ${ }^{36}$ in Fig. 5, the remaining particle $\mathrm{C}_{78} \mathrm{H}_{50}$ could possibly be charged with more electrons after the desorption of hydrogen atoms. Our calculation here is consistent with experiments showing that the negatively charged clusters are likely to remain with less hydrogen adsorption. ${ }^{13,38,39}$ Also, results in this work support our earlier assumption that the formation of diamond particles is independent of hydrogen pressure and those diamond particles are negatively charged. $5,6,13$

\section{SUMMARY}

In this work, we have studied the energetics of the charged diamond nanocluster through the first-principles pseudopotential calculations. In the charge-neutral cases, the outer surface of the diamond cluster reconstructs into a fullerene-like structure. However, in negatively charged cases, excess electrons saturate and stabilize the dangling bonds at the surface and the reconstruction is substantially reduced. In addition, the $\mathrm{C}-\mathrm{H}$ bond at the hydrogenated surfaces is shown to be destabilized upon a negative charging. Our results suggest that a negative charging could promote the growth of low pressure diamond, overcoming the surface stabilization by hydrogenation as well as graphitization.

\section{ACKNOWLEDGMENTS}

N.P. and H.N. thank JAMSTEC for computational resources of the Earth Simulator. N.P. and J.I. acknowledge the support by CNNC and the supercomputers of KISTI. N.P. thanks Professor E. Osawa and D. Tománek for generous and helpful discussions.
*Email address: noejung.park@ samsung.com

${ }^{1}$ J.C. Angus and C.C. Hayman, Science 241, 913 (1988).

${ }^{2}$ W.A. Yarbrough and R. Messier, Science 247, 688 (1990).

${ }^{3}$ P.K. Bachmann, D. Leers, and H. Lydtin, Diamond Relat. Mater. 1, 1 (1991).

${ }^{4}$ K. Okano, S. Koizumi, S.R.P. Silva, and G.A.J. Amaratanga, Nature (London) 381, 140 (1996).

${ }^{5}$ A.V. Palnichenko, A.M. Jonas, J.-C. Charlier, A.S. Aronin, and J.-P. Issi, Nature (London) 402, 162 (1999).

${ }^{6}$ N.M. Hwang and D.Y. Kim, J. Cryst. Growth 218, 40 (2000).

${ }^{7}$ P. Badziag, W.S. Verwoerd, W.P. Ellis, and N.R. Greiner, Nature (London) 343, 244 (1999).
${ }^{8}$ W.R.L. Lambrecht, C.H. Lee, B. Segall, J.C. Angus, Z. Li, and M. Sunkara, Nature (London) 364, 607 (1993).

${ }^{9}$ For a review, see P.E. Pehrsson and J.E. Butler, in Diamond Films and Coatings, edited by R.D. Davis (Noyes, Park Ridge, NJ, 1993), pp. 68-146.

${ }^{10}$ N.W. Winter and F.H. Ree, J. Comput.-Aided Mater. Des. 5, 279 (1998).

${ }^{11}$ D.M. Gruen, S. Liu, A.R. Krauss, J. Luo, and X. Pan, Appl. Phys. Lett. 64, 1502 (1994).

${ }^{12}$ M. Yoshimoto, K. Yoshida, H. Maruta, Y. Hishitani, H. Koinuma, S. Nishio, M. Kakihana, and T. Tachibana, Nature (London) 399, 340 (1999). 
${ }^{13}$ I.D. Jeon, C.J. Park, D.Y. Kim, and N.M. Hwang, J. Cryst. Growth 213, 79 (2000).

${ }^{14}$ I. Gouzman, I. Lior, and A. Hoffman, Appl. Phys. Lett. 72, 296 (1998)

${ }^{15}$ C. Sun, W.J. Zhang, N. Wang, C.Y. Chan, I. Bello, C.S. Lee, and S.T. Lee, J. Appl. Phys. 88, 3354 (2000).

${ }^{16}$ X.T. Zhou, Q. Li, F.Y. Meng, I. Bello, C.S. Lee, S.T. Lee, and Y. Liftshitz, Appl. Phys. Lett. 80, 3307 (2002).

${ }^{17}$ P. Hohenberg and W. Kohn, Phys. Rev. 136, B864 (1964); W. Kohn and L.J. Sham, Phys. Rev. 140, A1133 (1965).

${ }^{18}$ N. Troullier and J.L. Martins, Phys. Rev. B 43, 1993 (1991).

${ }^{19}$ L. Kleinman and D.M. Bylander, Phys. Rev. Lett. 48, 1425 (1982).

${ }^{20}$ D.M. Ceperley and B.J. Alder, Phys. Rev. Lett. 45, 566 (1980).

${ }^{21}$ J.P. Perdew and A. Zunger, Phys. Rev. B 23, 5048 (1981).

${ }^{22}$ J. Ihm, A. Zunger, and M.L. Cohen, J. Phys. C 12, 4409 (1979).

${ }^{23}$ G. Onida, L. Reining, R.W. Godby, R.D. Sole, and W. Andreoni, Phys. Rev. Lett. 75, 818 (1995).

${ }^{24}$ M.R. Jarvis, I.D. White, R.W. Godby, and M.C. Payne, Phys. Rev. B 56, 14972 (1997).

${ }^{25}$ N. Park, K. Lee, S. Han, J. Yu, and Jisoon Ihm, Phys. Rev. B 65 , 121405 (2002).

${ }^{26}$ O.F. Sankey and D.J. Niklewski, Phys. Rev. B 40, 3979 (1989).

${ }^{27}$ G. Makov and M.C. Payne, Phys. Rev. B 51, 4014 (1995).
${ }^{28}$ S.D. Kenny, A.P. Horsfield, and H. Fujitani, Phys. Rev. B 62, 4899 (2000).

${ }^{29}$ M. Scheffler, J.P. Vigneron, and G.B. Bachelet, Phys. Rev. B 31, 6541 (1985).

${ }^{30}$ J.-Y. Raty, G. Galli, C. Bostedt, T.W. van Buuren, and L.J. Terminello, Phys. Rev. Lett. 90, 037401 (2003).

${ }^{31}$ V.L. Kuznetsov, I.L. Zilberberg, Yu.V. Butenko, A.L. Chuvilin, and B. Segall, J. Appl. Phys. 86, 863 (1999).

${ }^{32}$ V.V. Roddatis, V.L. Kuznetsov, Yu.V. Butenko, D.S. Su, and R. Schlögl, Phys. Chem. Chem. Phys. 4, 1964 (2002).

${ }^{33}$ Note that if the energy of irradiating electron is high enough $(\geqslant 200 \mathrm{keV})$, it induces an inverse transformation from graphitic onions to diamond, which is well explained by F. Banhart, Rep. Prog. Phys. 62, 1181 (1999).

${ }^{34}$ J.E. Dahl, S.G. Liu, and R.M.K. Carlson, Science 299, 96 (2003).

${ }^{35}$ I. Efremenko, M. Sheintuch, I. Gouzman, and A. Hoffman, J. Cryst. Growth 198/199, 951 (1999).

${ }^{36}$ A. Y. Lozovoi and A. Alavi, Phys. Rev. B 68, 245416 (2003).

${ }^{37}$ P. Keblinski, S.K. Nayak, P. Zapol, and P.M. Ajayan, Phys. Rev. Lett. 89, 255503 (2002).

${ }^{38} \mathrm{Ph}$. Gerhardt, S. Loffler, and K.H. Homann, Chem. Phys. Lett. 137, 306 (1987).

${ }^{39}$ I.D. Jeon, C.J. Park, D.Y. Kim, and N.M. Hwang, J. Cryst. Growth 223, 6 (2001). 